\title{
Evaluation of the Efficiency of Two Different Freezing Media and Two Different Protocols to Preserve Human Spermatozoa from Cryoinjury
}

\author{
Gemma Fabozzi, Maria Flavia Starita, Emilia Rega, Alessandra Alteri, \\ Antonio Colicchia, Claudio Piscitelli, and Pierluigi Giannini
}

FertiClinic, Casa di Cura Villa Margherita, Viale di Villa Massimo 48, 00161 Rome, Italy

Correspondence should be addressed to Gemma Fabozzi; gemmafabozzi@hotmail.it

Received 21 April 2016; Accepted 21 June 2016

Academic Editor: Yuksel Agca

Copyright (C) 2016 Gemma Fabozzi et al. This is an open access article distributed under the Creative Commons Attribution License, which permits unrestricted use, distribution, and reproduction in any medium, provided the original work is properly cited.

It is universally recognized that cryopreservation impairs sperm quality. In order to improve postthawing sperm survival and motility, media of different composition and different protocols have been proposed. However, no clear evidence is available to understand which are the most efficient protocol and medium for sperm cryopreservation. The present study evaluates the efficiency of two different cryopreservation protocols and two common freezing media (FM) containing different cryoprotectants (CPs), TEST Yolk Buffer (TYB) and Sperm Freeze (SF), to preserve human sperm quality. Our data suggest that TYB is better than SF both in terms of postthaw viability and in terms of progressive motility, while the direct addition of FM to the sperm sample resulted in the most efficient protocol in terms of postthaw viability but not in terms of progressive motility.

\section{Introduction}

Sperm cryopreservation has long been used in the clinical practice of assisted reproduction to manage male infertility and to store donor samples or as a tool in the livestock industry. The main goal of male gamete cryopreservation is to preserve sperm viability, motility, and fertilizing ability; however, it has been largely reported in the literature that the freezingthawing procedures cause severe structural and functional damage to spermatozoa, impairing cell membranes [1-3] and sperm motility $[4,5]$. This is due to physical stresses to which cells are usually exposed during freezing: the direct effects of reduced temperature and physical changes associated with ice formation [6]. Furthermore, sperm membrane composition differs from the other cell membranes in the relative proportion of lipid species: there is high proportion of ether-linked fatty acids and phospholipidic hexaenoic acid side chains which contribute to increasing membrane fluidity, while sterols like cholesterol counteract this potential instability [7, 8]. At physiological temperatures, sperm membranes coexist in two phases, fluid and gel, but as the temperature is lowered, a phase transition occurs in favor of the gel form [9]. When cryopreservation is performed, this phase transition leads to a reduction in membrane fluidity, which has been associated with lower sperm survival and motility [10]. Cryoprotectants are hyperosmotic additives which preserve cells by stabilizing intracellular proteins by reducing intracellular ice formation and by moderating the effect of concentrated intracellular and extracellular electrolytes [11]. The first cryoprotectant to cryopreserved spermatozoa was glycerol [12] and it remains the most widely used, generally at a concentration of $10-15 \%$ [10, 13-15]. However, other cryoprotectant media have been formulated, such as TEST Yolk and HSPM, both of which contain glycine and glucose $[15,16]$.

Different protocols for sperm cryopreservation have been defined to improve sperm survival after thawing. Most of them foresee sperm washing prior to freezing; however, Grizard and colleagues [17] reported that the presence of seminal plasma in the cryopreservation medium improved sperm survival. Some groups have suggested different strategies to improve sperm quality when freezing-thawing is performed. For example, Esteves and colleagues [18] speculated that the removal of immotile and damaged sperm prior to cryopreservation may be beneficial, so they used the swim-up method 


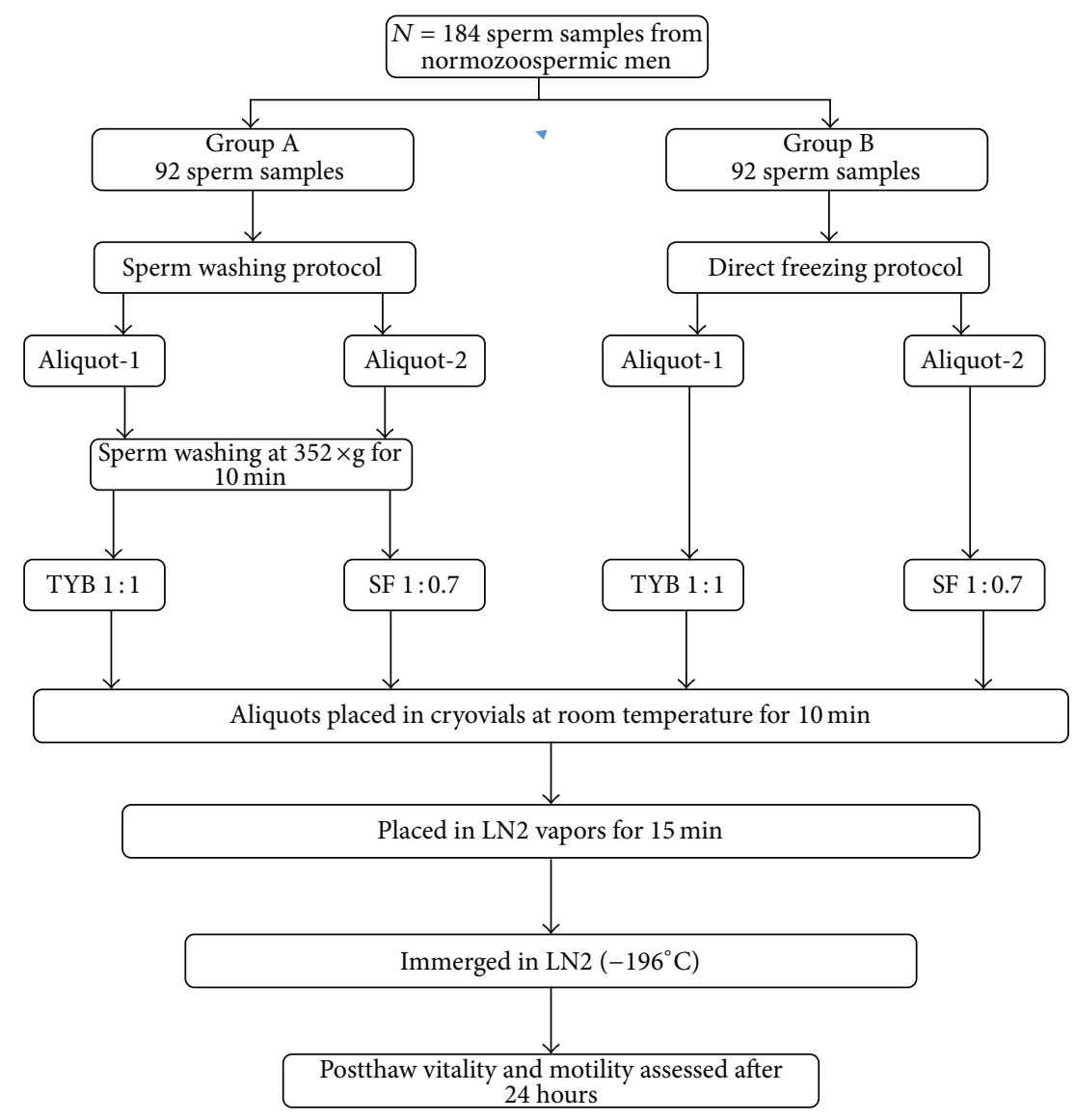

FIGURE 1: Flow chart of study design.

prior to freezing to select a subpopulation of highly motile sperm. Oehninger and colleagues [19] proposed the use of stimulants like pentoxifylline to increase motility.

Although different studies concerning semen cryopreservation are reported in the literature, no clear evidence is available to understand which are the most efficient protocol and FM for sperm cryopreservation. The aim of this study was to evaluate the efficiency of two common FM, TEST Yolk Buffer (TYB) and Sperm Freeze (SF), and two different freezing protocols, one entailing a sperm washing procedure and one not, to preserve sperm quality in terms of cryosurvival and postthaw motility.

\section{Materials and Methods}

2.1. Study Design. This is a prospective cohort study. Semen samples were obtained from 184 normozoospermic patients following sperm evaluation performed at FertiClinic, Villa Margherita, from September to December 2014. Patients were randomized in two groups: group $\mathrm{A}(N=92$ samples $)$ and group $\mathrm{B}(\mathrm{N}=92$ samples). Samples in group A were split into 2 aliquots and cryopreserved adding, respectively, TEST Yolk Buffer (Irvine Scientific, California) and Sperm Freeze (FertiPro, Belgium) after washing and resuspension. On the contrary, group B samples were split into 2 aliquots and cryopreserved adding, respectively, TYB and SF directly to the semen (Figure 1). Sperm viability and progressive motility were the outcome measures assessed after thawing by a blinded observer. A single team of biologists coordinated all biological work, ensuring that both freezing protocols and viability and motility assessment were standardized.

2.2. Semen Analysis. Sperm samples were collected by masturbation after 3-5 days of sexual abstinence and examined by microscopy at 40x magnification after liquefaction. Sperm concentration was assessed using a Makler counting chamber; motility was assessed according to World Health Organization criteria 2010 [20] both before and after cryopreservation.

2.3. Comparison between the Two Cryopreservation Methods. In group $\mathrm{A}, 1 \mathrm{~mL}$ of the sperm sample was divided into two aliquots of $0.5 \mathrm{~mL}$ and placed into two different tubes containing $4 \mathrm{~mL}$ sperm washing medium (sperm washing protocol). Aliquots were then centrifuged at $352 \times \mathrm{g}$ for 10 minutes and each sperm pellet was resuspended with $0.5 \mathrm{~mL}$ of HEPES; then, TYB was added to aliquot $\mathrm{A} 1$ at a final ratio of $1: 1(\mathrm{v} / \mathrm{v})$, while SF was added dropwise to aliquot A2 at a final ratio of $1: 0.7(\mathrm{v} / \mathrm{v})$.

In group $\mathrm{B}, 1 \mathrm{~mL}$ of the sperm sample was divided into two aliquots of $0.5 \mathrm{~mL}$ and placed into two different tubes; then, FM were added directly to the aliquots (direct freezing 
TABLE 1: Postthaw viability and progressive motility of semen samples cryopreserved by two different freezing protocols using two different FM.

\begin{tabular}{|c|c|c|c|c|c|c|c|c|}
\hline \multirow{2}{*}{ Variable } & \multicolumn{2}{|c|}{ Group A (sperm washing protocol) } & \multicolumn{2}{|c|}{ Group B (direct freezing protocol) } & \multicolumn{4}{|c|}{$P$ value } \\
\hline & TYB & SF & TYB & SF & $P^{\mathrm{a}}$ & $P^{\mathrm{b}}$ & $P^{c}$ & $P^{\mathrm{d}}$ \\
\hline Viability (\%) & $21.20 \pm 1$ & $15.62 \pm 0.71$ & $27.21 \pm 1.69$ & $21.71 \pm 1.60$ & $<0.0001$ & $<0.0001$ & 0.006 & 0.0002 \\
\hline Progressive motility (\%) & $18.41 \pm 1.03$ & $13.99 \pm 0.87$ & $16.63 \pm 1.67$ & $13.38 \pm 1.46$ & $<0.0001$ & 0.005 & NS & NS \\
\hline
\end{tabular}

Note: values are mean \pm SEM. TYB, TEST Yolk Buffer; SF, Sperm Freeze.

$P^{\mathrm{a}}$, paired $t$-test for TYB versus SF in group $\mathrm{A}$.

$P^{\mathrm{b}}$, paired $t$-test for TYB versus SF in group B.

$P^{\mathrm{c}}$, unpaired $t$-test for TYB washed versus TYB not washed.

$P^{\mathrm{d}}$, unpaired $t$-test for SF washed versus SF not washed.

protocol): TYB was added to $\mathrm{A} 1$ at a final ratio of $1: 1(\mathrm{v} / \mathrm{v})$, while SF was added to aliquot $\mathrm{A} 2$ at a final ratio of $1: 0.7(\mathrm{v} / \mathrm{v})$.

In both cases, FM were added in a dropwise manner, gently mixed, and then placed into cryovials at room temperature for 10 minutes to allow for proper equilibration between the cells and the medium. Then, samples were placed in nitrogen vapors (between $-80^{\circ} \mathrm{C}$ and $-100^{\circ} \mathrm{C}$ ) for 15 minutes and finally immerged in liquid nitrogen at $-196^{\circ} \mathrm{C}$.

2.4. Thawing Procedure. The cryopreserved aliquots from both groups were thawed in an identical manner. The cryovial was kept at RT for 10 minutes; then freezing medium was removed by adding $4 \mathrm{~mL}$ of sperm washing medium washing the sample in the centrifuge at $352 \times \mathrm{g}$ for 10 minutes. Subsequently, the sperm pellet was resuspended with $250 \mu \mathrm{L}$ of HEPES: $50 \mu \mathrm{L}$ was immediately processed for viability, while the rest of the sample was left at $37^{\circ} \mathrm{C}$ and sperm motility was assessed after 30 minutes by a blinded observer at $37^{\circ} \mathrm{C}$.

2.5. Viability Assessment. Sperm postthaw viability was assessed using the VitalScreen ${ }^{\mathrm{TM}}$ kit (FertiPro, Belgium) which uses the eosin-nigrosin staining technique to establish the percentage of live spermatozoa assessing the membrane integrity of the cells [20]. The technique is based on the principle that dead cells absorb eosin and as a result stain red. The nigrosin provides a dark background which makes it easier to assess the slides. Briefly, a $50 \mu \mathrm{L}$ drop of thawed semen was mixed with two drops of $1 \%$ eosin stain. After $30 \mathrm{~s}$, three drops of $5 \%$ nigrosin were added and the solutions mixed. A $10 \mu \mathrm{L}$ drop of this mixture was placed onto a microscope slide and a smear was made. The smears were allowed to air dry and were viewed under oil immersion with light microscopy at a magnification of 100x. Live sperm appeared white, while dead sperm with disrupted membranes had absorbed the eosin stain and appeared red. Sperm viability was quantified according to $\mathrm{WHO}$ guidelines by counting 200 sperm and expressed as percentage live sperm.

2.6. Ethics. All participants enrolled in the study filled out a written informed consent in which they expressed that they consent to donate their sperm for research purposes. The FM and protocols used to preserve sperm samples in this study are routinely used by different IVF units all over the world, so institutional review board was not required.
2.7. Statistics. Paired two-tailed Student's $t$-test was used to compare the two FM and the unpaired Student's $t$-test was used to compare the two cryopreservation protocols. Results are given as mean and standard error of the mean. Differences were considered significant at $P<0.05$. Stepwise linear regression (exit value, $P=0.1$ ) was performed to adjust for male age and sperm quality (volume, concentration, morphology, and motility) as possible confounding factors when the two cryopreservation protocols were compared. $\beta$ regression and the $95 \%$ confidence interval (CI) were estimated. SPSS Statistic 21 software was used to perform statistical analysis.

\section{Results}

Semen samples cryopreserved with TYB resulted in better postthaw viability with respect to SF when aliquots were washed and resuspended (Group A) or for the aliquots cryopreserved without washing (Group B). Samples cryopreserved using TYB also showed a better progressive motility in both groups (Table 1).

Then, the freezing protocol was investigated. Sperm viability was significantly higher for samples cryopreserved using direct freezing protocol (Group B) either when TYB was used as freezing medium or when SF was used (Table 1). This result was confirmed even when adjusted for male age and sperm quality, independently from the FM used $(\beta=$ 5.66, CI 95\%: $1.8-9.5$, and $P=0.004$ for TYB and $\beta=5.8$, CI 95\%: 2.9-8.8, and $P<0.001$ for SF). No significant difference was detected with regard to postthaw progressive motility comparing the freezing protocols either when TYB was used or when SF was used (Table 1 ). The average motility $\%$ change is reported in Table 2 .

\section{Discussion}

The aim of this study was to compare the efficiency of two freezing media and two cryopreservation methods to protect sperm quality in terms of cryosurvival and postthaw motility. It is well known that cryoprotectants contained in FM protect cells from cryodamage; however, their toxicity is largely unknown [21, 22]. Stanic and colleagues [21] reported that most of the decrease in sperm motility during cryopreservation can be due to exposure to CPs rather than to the freezing process. Thus, the choice of FM represents a crucial point in 
TABLE 2: Progressive motility values and \% change before and after semen samples cryopreservation using two different freezing protocols and two different FM.

\begin{tabular}{|c|c|c|c|c|c|c|c|c|}
\hline \multirow{2}{*}{ Progressive motility (\%) } & \multicolumn{2}{|c|}{ Group A (sperm washing protocol) } & \multicolumn{2}{|c|}{ Group B (direct freezing protocol) } & \multicolumn{4}{|c|}{$P$ value } \\
\hline & TYB & SF & TYB & SF & $P^{\mathrm{a}}$ & $P^{\mathrm{b}}$ & $P^{\mathrm{c}}$ & $P^{\mathrm{d}}$ \\
\hline Before & \multicolumn{2}{|c|}{$39.10 \pm 13.58$} & \multicolumn{2}{|c|}{$41.08 \pm 14.2$} & & & & \\
\hline After & $18.41 \pm 1.03$ & $13.99 \pm 0.87$ & $16.63 \pm 1.67$ & $13.38 \pm 1.46$ & & & & \\
\hline$\%$ change & $52.19 \pm 2.76$ & $63.81 \pm 2.37$ & $58.44 \pm 3.82$ & $65.97 \pm 3.66$ & $<0.001$ & 0.0114 & NS & NS \\
\hline
\end{tabular}

Note: values are mean \pm SEM. TYB, TEST Yolk Buffer; SF, Sperm Freeze.

$P^{\mathrm{a}}$, paired $t$-test for TYB versus SF in group A.

$P^{\mathrm{b}}$, paired $t$-test for TYB versus $\mathrm{SF}$ in group B.

$P^{\mathrm{c}}$, unpaired $t$-test for TYB washed versus TYB not washed.

$P^{\mathrm{d}}$, unpaired $t$-test for SF washed versus SF not washed.

cryopreservation procedures. Our results showed significant postthaw viability and progressive motility for samples cryopreserved using TYB with respect to SF, independently from the cryopreservation method used. Our findings are consistent with other studies demonstrating that TYB results in better recovery of motile sperm [21, 23-25]; however, we also investigated the effect on postthaw viability and provided a larger sample size.

The mechanism by which TYB functions is unclear. Each freezing medium is composed by a combination of penetrating osmolytes, which stabilize intracellular proteins, reduce the temperature at which intracellular ice forms, and decrease the impact of intracellular and extracellular electrolytes inside the cell, and nonpenetrating osmolytes, which act as osmotic buffers to protect against cell swelling during the addition/removal of cryoprotectants [22]. Since TYB and SF are composed of the same permeating cryoprotectants (glycerol), probably the nonpermeating component makes the difference: SF contains albumin, while TYB contains egg yolk, which is mainly composed of lipoproteins, phospholipids, and cholesterol. Probably, the low-density lipoprotein fraction of egg yolk, rich in phospholipids, allows for the maintenance of major sperm membrane fluidity, since it permits a greater lipid exchange and contrasts the physiological phase transition in favor of the gel form which normally occurs when the temperature is lowered [9]. On the contrary, albumin contained in SF probably does not succeed in maintaining such fluidity, leading to a lower sperm motility and survival after thawing [10]. Thus, the low-density lipoprotein fraction of egg yolk present in TYB probably exerts a more powerful effect than albumin present in SF, acting as osmotic buffers and protecting the cell against cold shock injury.

The second part of our study was aimed at comparing the ability of two different cryopreservation methods in preserving sperm viability and motility. Data showed that the direct addition of FM to sperm samples resulted in the most efficient strategy in terms of postthaw viability, independently from the FM used. This result can be addressed to the presence of seminal plasma (SP). Its components, in fact, have been shown to be important modulators of sperm function. Notably, animal studies have highlighted that SP proteins seem to inhibit capacitation, lipid peroxidation, and cold shock injury [26-28]. Furthermore, it has been shown that $\mathrm{SP}$ is able to protect membrane integrity in boar, ram, and bull sperm [29]. In human as well, it seems to reduce the deleterious effects of cryopreservation, as reported by Grizard and collegues [17]; however, their studies were conducted in free-medium biological material which did not offer adequate cryoprotection of samples.

An additional important aspect is that somatic cells are protected from oxidative stress by antioxidants present within their cytoplasm (superoxide dismutase, catalase, glutathione peroxidase, glutathione, vitamin $\mathrm{E}$, and vitamin C), whereas sperm cells lack a potent intracellular ROS defense system [30], since they lose most of their cytoplasm during their maturation process. However, seminal plasma is well endowed with antioxidant buffer capacity as already demonstrated by different groups [31-33] and all the internal natural antioxidant molecules within SP, such as prostasomes, [34], acetylcarnitine/carnitine, or super oxide dismutase and catalase [35], provide the main sperm protection against oxidative stress.

Comparing the ability of the two different cryopreservation methods in preserving sperm viability and motility, no statistically significant difference was observed in terms of progressive motility, independently from the FM used. This result is in contrast with data reported by Petyim and colleagues [36] who observed a statistically significant difference with respect to progressive motility between samples processed for swim-up prior to cryopreservation and samples processed following cryopreservation: progressive motility was higher in the prepreparation group. This difference can be addressed to the fact that our study included only normozoospermic samples, while the study of Petyim and colleagues [36] included semen samples from infertile males and it has been shown that the quality of semen sample can be related to the outcome of cryopreservation. For example, the presence of dead spermatozoa, cellular debris, bacteria, or leukocytes detrimentally affects sperm survival and the fertility potential after thawing through ROS generation process [37]. In such cases, sperm preparation with swimup technique is indicated before freezing since it displays an advantage in apoptosis prevention and the stress caused by the cryopreservation procedure does not add to damage caused by free oxygen radicals.

According to authors, results obtained in the presented study can be of significant importance for clinical use, since they can provide advice concerning the best strategy to cryopreserve sperm samples. However, since some studies 
have shown the seminal plasma of infertile men to have an impaired nonenzymatic antioxidant capacity $[31,32]$ and others found an erratic distribution between the fertile and nonfertile populations [33], a new prospective study with the same design as this but enrolling pathological samples is in progress in our laboratory. The aim is to obtain a wider range of data and to understand if sperm freezing in the presence of seminal plasma has to be preferred also for these patients or if sperm preparation before freezing or media supplementation with antioxidants can be necessary in such cases. Further researches are always required to improve our knowledge and, in this case, to hopefully yield a universal technique for the cryopreservation of spermatozoa.

\section{Disclosure}

Preliminary data were presented at the ESHRE 31st annual meeting, Lisbon, Portugal, 14 to 17 June 2015.

\section{Competing Interests}

Gemma Fabozzi declares that there are no competing interests regarding the publication of this paper.

\section{References}

[1] G. M. Centola, R. F. Raubertas, and J. H. Mattox, "Cryopreservation of human semen. Comparison of cryopreservatives, sources of variability, and prediction of post-thaw survival," Journal of Andrology, vol. 13, no. 3, pp. 283-288, 1992.

[2] M. A. Richter, R. V. Haning Jr., and S. S. Shapiro, "Artificial donor insemination: fresh versus frozen semen: the patient as her own control," Fertility and Sterility, vol. 41, no. 2, pp. 277280, 1984.

[3] A. W. Y. Wong, P. C. Ho, M. Kwan, and H. K. Ma, "Factors affecting the success of artificial insemination by frozen donor semen," International Journal of Fertility, vol. 34, no. 1, pp. 2529, 1989.

[4] J. K. Critser, A. R. Huse-Benda, D. V. Aaker, B. W. Arneson, and G. D. Ball, "Cryopreservation of human spermatozoa. III. The effect of cryoprotectants on motility," Fertility and Sterility, vol. 50, no. 2, pp. 314-320, 1988.

[5] G. Verheyen, I. Pletincx, and A. Van Steirteghem, "Effect of freezing method, thawing temperature and post-thaw dilution/washing on motility (CASA) and morphology characteristics of high-quality human sperm," Human Reproduction, vol. 8, no. 10, pp. 1678-1684, 1993.

[6] P. Mazur, "Freezing of living cells: mechanisms and implications," The American Journal of Physiology, vol. 247, no. 3, pp. 125-142, 1984.

[7] W. V. Holt, "Fundamental aspects of sperm cryobiology: the importance of species and individual differences," Theriogenology, vol. 53, no. 1, pp. 47-58, 2000.

[8] J. E. Parks, "Hypothermia and mammalian gametes," in Reproductive Tissue Banking_Scientific Principles, A. Karow and J. K. Critser, Eds., Academic Press, San Diego, Calif, USA, 1997.

[9] W. V. Holt, "Basic aspects of frozen storage of semen," Animal Reproduction Science, vol. 62, no. 1-3, pp. 3-22, 2000.

[10] M. N. Giraud, C. Motta, D. Boucher, and G. Grizard, "Membrane fluidity predicts the outcome of cryopreservation of human spermatozoa," Human Reproduction, vol. 15, no. 10, pp. 2160-2164, 2000.

[11] P. Mazur, "Kinetics of water loss from cells at subzero temperatures and the likelihood of intracellular freezing," The Journal of General Physiology, vol. 47, pp. 347-369, 1963.

[12] C. Polge, A. U. Smith, and A. S. Parkes, "Revival of spermatozoa after vitrification and dehydration at low temperatures," Nature, vol. 164, no. 4172, pp. 666-676, 1949.

[13] R. V. Devireddy, D. J. Swanlund, K. P. Roberts, J. L. Pryor, and J. C. Bischof, "The effect of extracellular ice and cryoprotective agents on the water permeability parameters of human sperm plasma membrane during freezing," Human Reproduction, vol. 15, no. 5, pp. 1125-1135, 2000.

[14] C. Srisombut, M. Morshedi, M. H. Lin, A. Nassar, and S. Oehninger, "Comparison of various methods of processing human cryopreserved-thawed semen samples," Human Reproduction, vol. 13, no. 8, pp. 2151-2157, 1998.

[15] G. J. Morris, E. Acton, and S. Avery, "A novel approach to sperm cryopreservation," Human Reproduction, vol. 14, no. 4, pp. 1013$1021,1999$.

[16] M. E. Hammadeh, A. S. Askari, T. Georg, P. Rosenbaum, and W. Schmidt, "Effect of freeze-thawing procedure on chromatin stability, morphological alteration and membrane integrity of human spermatozoa in fertile and subfertile men," International Journal of Andrology, vol. 22, no. 3, pp. 155-162, 1999.

[17] G. Grizard, V. Chevalier, J. F. Griveau, D. Le Lannou, and D. Boucher, "Influence of seminal plasma on cryopreservation of human spermatozoa in a biological material-free medium: study of normal and low-duality semen," International Journal of Andrology, vol. 22, no. 3, pp. 190-196, 1999.

[18] S. C. Esteves, R. K. Sharma, A. J. Thomas Jr., and A. Agarwal, "Improvement in motion characteristics and acrosome status in cryopreserved human spermatozoa by swim-up processing before freezing," Human Reproduction, vol. 15, no. 10, pp. 21732179, 2000.

[19] S. Oehninger, N. K. Duru, C. Srisombut, and M. Morshedi, "Assessment of sperm cryodamage and strategies to improve outcome," Molecular and Cellular Endocrinology, vol. 169, no. 12, pp. 3-10, 2000.

[20] World Health Organization, Who Laboratory Manual for the Examination and Processing of Human Semen, World Health Organization, Geneva, Switzerland, 5th edition, 2010.

[21] P. Stanic, M. Tandara, Z. Sonicki, V. Simunic, B. Radakovic, and E. Suchanek, "Comparison of protective media and freezing techniques for cryopreservation of human semen," European Journal of Obstetrics \& Gynecology and Reproductive Biology, vol. 91, no. 1, pp. 65-70, 2000.

[22] H. M. Picton, S. S. Kim, and R. G. Gosden, "Cryopreservation of gonadal tissue and cells," British Medical Bulletin, vol. 56, no. 3, pp. 603-615, 2000.

[23] G. Ragni, G. C. Lombroso Finzi, A. Caccamo, A. Dalla Serra, and P. G. Crosignani, "Enhanced quality of capacitated spermatozoa from oligoasthenozoospermic men after incubation in TEST-yolk medium," Andrologia, vol. 25, no. 6, pp. 337-339, 1993.

[24] M. E. Hammadeh, S. Greiner, P. Rosenbaum, and W. Schmidt, "Comparison between human sperm preservation medium and TEST-yolk buffer on protecting chromatin and morphology integrity of human spermatozoa in fertile and subfertile men after freeze-thawing procedure," Journal of Andrology, vol. 22, no. 6, pp. 1012-1018, 2001. 
[25] L. S. McGonagle, M. Goldstein, J. Feldschuh, and R. H. Foote, "The influence of cryoprotective media and processing procedures on motility and migration of frozen-thawed human sperm," Asian Journal of Andrology, vol. 4, no. 2, pp. 137-141, 2002.

[26] Y.-H. Huang, S.-T. Chu, and Y.-H. Chen, "A seminal vesicle autoantigen of mouse is able to suppress sperm capacitationrelated events stimulated by serum albumin," Biology of Reproduction, vol. 63, no. 5, pp. 1562-1566, 2000.

[27] R. Pérez-Pé, J. A. Cebrián-Pérez, and T. Muio-Blanco, "Semen plasma proteins prevent cold-shock membrane damage to ram spermatozoa," Theriogenology, vol. 56, no. 3, pp. 425-434, 2001.

[28] C. Schöneck, J. Braun, and R. Einspanier, "Sperm viability is influenced in vitro by the bovine seminal protein aSFP: effects on motility, mitochondrial activity and lipid peroxidation," Theriogenology, vol. 45, no. 3, pp. 633-642, 1996.

[29] W. M. C. Maxwell and L. A. Johnson, "Physiology of spermatozoa at high dilution rates: the influence of seminal plasma," Theriogenology, vol. 52, no. 8, pp. 1353-1362, 1999.

[30] J. Aitken and H. Fisher, "Reactive oxygen species generation and human spermatozoa: the balance of benefit and risk," BioEssays, vol. 16, no. 4, pp. 259-267, 1994.

[31] S. E. M. Lewis, P. M. Boyle, K. A. McKinney, I. S. Young, and W. Thompson, "Total antioxidant capacity of seminal plasma is different in fertile and infertile men," Fertility and Sterility, vol. 64, no. 4, pp. 868-870, 1995.

[32] R. Smith, D. Vantman, J. Ponce, J. Escobar, and E. Lissi, "Total antioxidant capacity of human seminal plasma," Human Reproduction, vol. 11, no. 8, pp. 1655-1660, 1996.

[33] M. Jóźwik, M. Jóźwik, W. Kuczyński, and M. Szamatowicz, "Nonenzymatic antioxidant activity of human seminal plasma," Fertility and Sterility, vol. 68, no. 1, pp. 154-157, 1997.

[34] F. G. Kravets, J. Lee, B. Singh, A. Trocchia, S. N. Pentyala, and S. A. Khan, "Prostasomes: current concepts," Prostate, vol. 43, no. 3, pp. 169-174, 2000.

[35] S. E. M. Lewis, E. S. L. Sterling, I. S. Young, and W. Thompson, "Comparison of individual antioxidants of sperm and seminal plasma in fertile and infertile men," Fertility and Sterility, vol. 67, no. 1, pp. 142-147, 1997.

[36] S. Petyim, C. Neungton, I. Thanaboonyawat, P. Laokirkkiat, and R. Choavaratana, "Sperm preparation before freezing improves sperm motility and reduces apoptosis in post-freezing-thawing sperm compared with post-thawing sperm preparation," Journal of Assisted Reproduction and Genetics, vol. 31, no. 12, pp. 1673-1680, 2014.

[37] L. K. Thomson, S. D. Fleming, R. J. Aitken, G. N. De Iuliis, J.-A. Zieschang, and A. M. Clark, "Cryopreservation-induced human sperm DNA damage is predominantly mediated by oxidative stress rather than apoptosis," Human Reproduction, vol. 24, no. 9, pp. 2061-2070, 2009. 


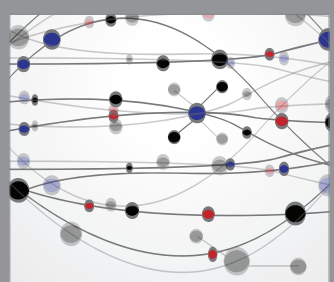

The Scientific World Journal
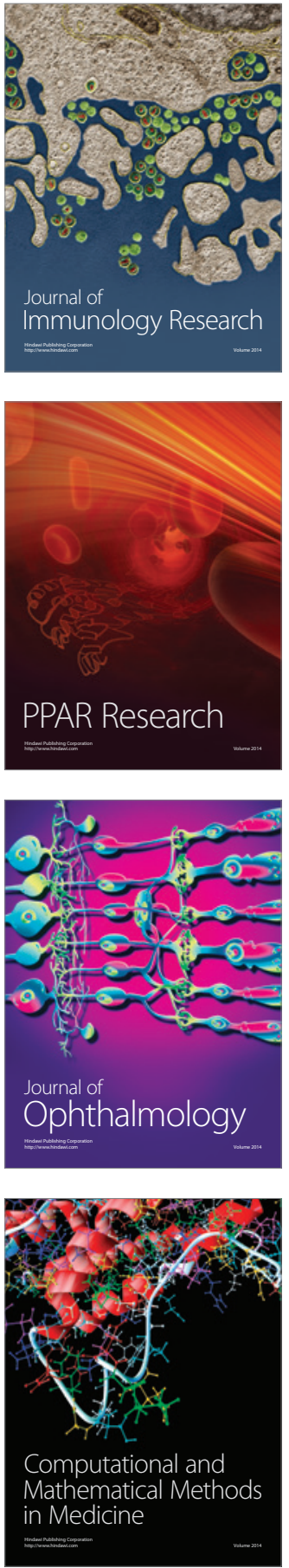

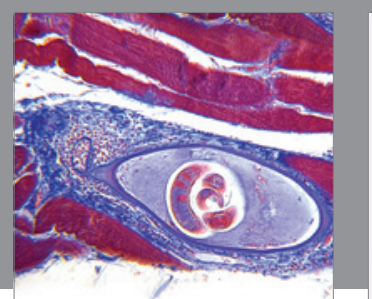

Gastroenterology Research and Practice

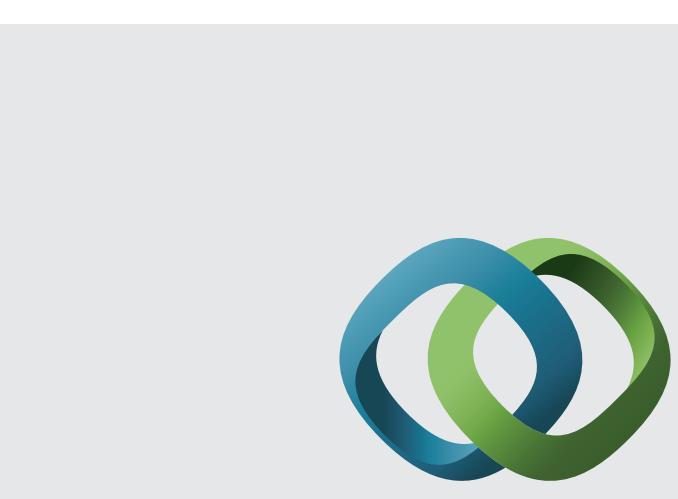

\section{Hindawi}

Submit your manuscripts at

http://www.hindawi.com
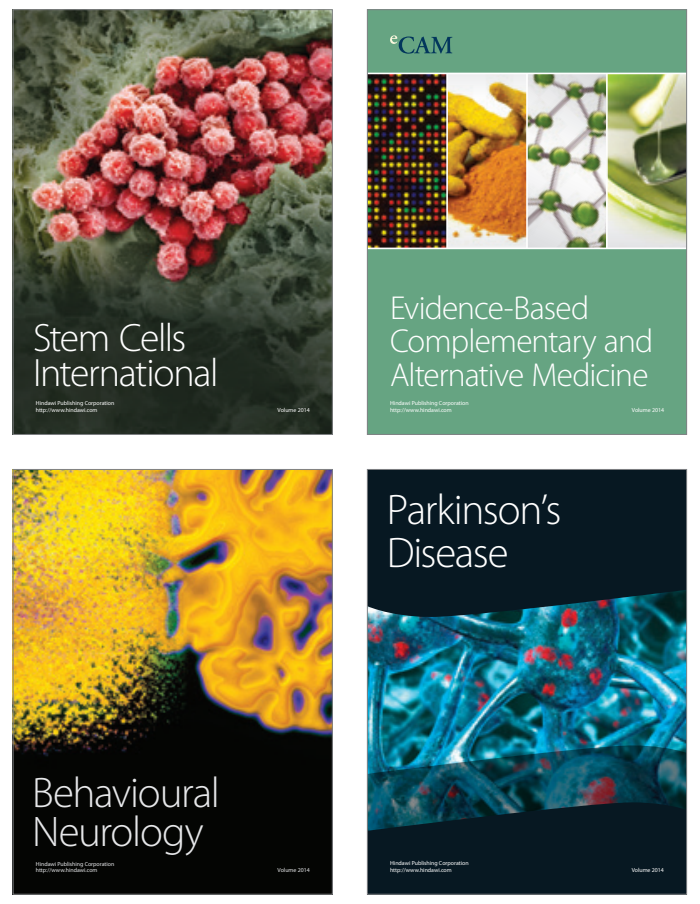
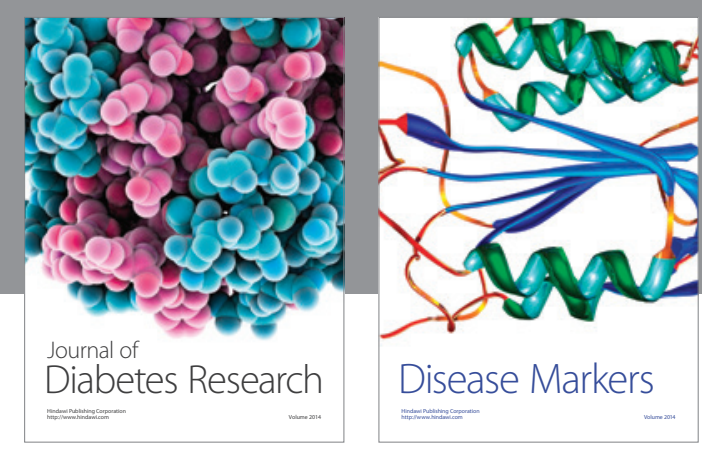

Disease Markers
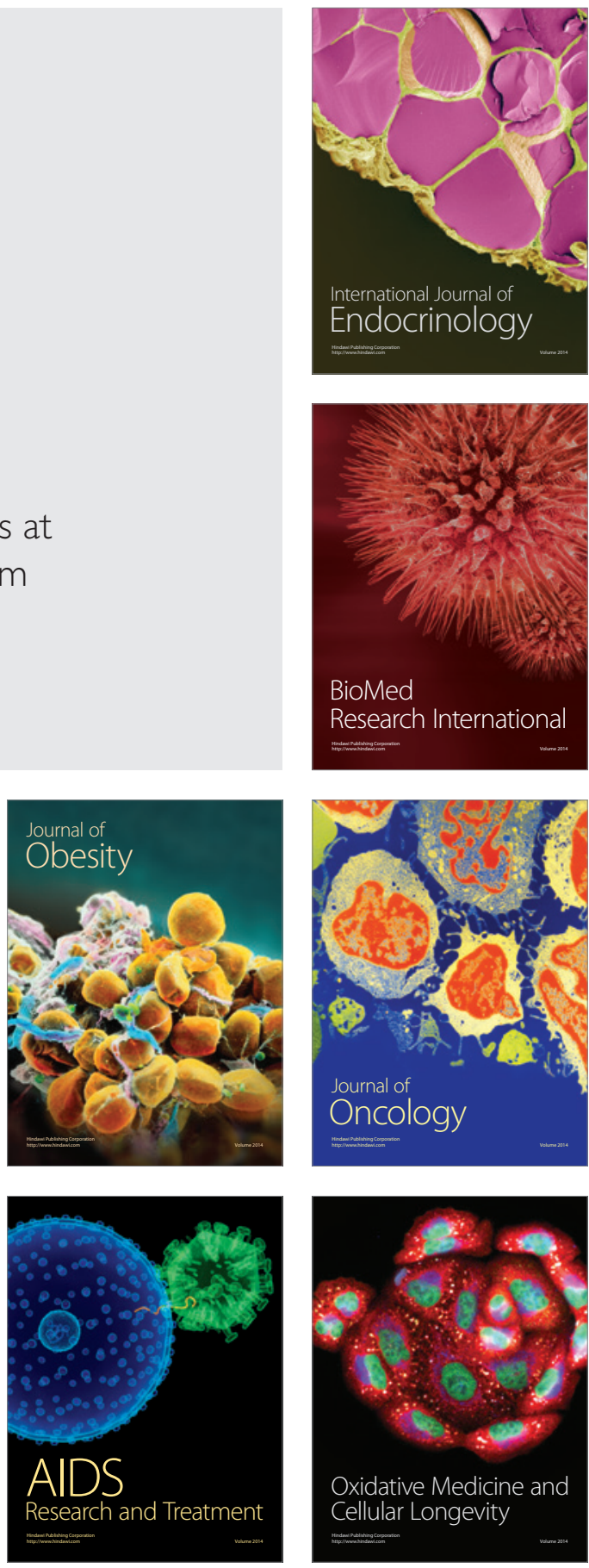